\title{
Basic Study for New Assistive Technology Based on Brain Activity during Car Driving
}

\author{
Hiroaki Inoue \\ Tokyo University of Science, Suwa \\ Research course of Engineering/Management \\ Chino-city, Japan
}

\author{
Shunji Shimizu, Noboru Takahashi and Yasuhito \\ Yoshizawa \\ Tokyo University of Science, Suwa \\ Department of Computer Engineering \\ Chino-city, Japan
}

\author{
Hiroyuki Nara \\ Hokkaido University \\ Graduate School of Information Science and Technology \\ Sapporo-city, Japan
}

\author{
Fumikazu Miwakeichi \\ The Institute of Statistical Mathematics \\ Spatial and Time Series Modeling Group \\ Tachikawa-city, Japan \\ Nobuhide Hirai \\ Tokyo Medical and Dental University \\ Health Service Center \\ Bunkyou-ku, Japan
}
Senichiro Kikuchi, and Satoshi Kato
Jichi Medical University
Department of Psychiatry
Shimotsuke-city, Japan

\author{
Eiju Watanabe \\ Jichi Medical University \\ Department of Neurosurgery \\ Shimotsuke-city, Japan
}

\begin{abstract}
Recently, it is necessary to develop a new system which assists driving car and wheelchair as aged society. The final our purpose in this research is to contribute to developing of assistive robot and related-apparatus. In terms of developing a new system, we thought that it is important to examine behaviors as well as spatial recognition. Therefore, experiments have been performed for an examination of human spatial perceptions, especially right and left recognition, during car driving by using NIRS. In previous research, it has been documented that there were significant differences at dorsolateral prefrontal cortex at left hemisphere during virtual driving task and actual driving. In this paper, we performed measuring the brain activity during car driving by using NIRS. And we performed statistical analysis of the brain activity. The purpose of this paper is discovering the brain region which was involved in decision making when human drive a car and considering between human movement and brain activity during car driving.
\end{abstract}

Keywords-brain information processing during driving task; spatial cognitive task; determining direction; NIRS

\section{INTRODUCTION}

Human movements change relative to their environment. Nevertheless, human recognizes the new location and decides what behavior to take when they move by a vehicle. It is important to analyze the human spatial perception for developing autonomous robots or automatic driving. In the previous study, the relation of the theta brain waves to the human spatial perception was discussed in [1][2]. When humans perceive space, for example, try to decide the next action in a maze, the theta brain waves saliently appear. This means human have a searching behavior to find a goal at an unknown maze. From the side of human navigation, Maguire et al. measured the brain activations using complex virtual reality town [3]. But, every task is notional and the particulars about the mechanism that enables humans to perceive space and direction are yet unknown. Also, Brain activities concerned with cognitive tasks during car driving have been examined. For example, there was a report about brain activity when disturbances were given to subjects who manipulated a driving simulator. Also, power spectrums increased in beta and theta bands [4]. However, there is little report on the relationship among right and left perception and driving task.

So, we performed experiments in which perception tasks were required during virtual car driving using Near Infrared Spectroscopy (NIRS) [5]. From experimental results, there were significant differences at dorsolateral prefrontal cortex in left hemisphere via one-sample t-test when subjects watched driving movie and moving their hand in circles as if handling a steering wheel [6].

In addition, we performed experiments in real-space, which were performed by taking NIRS in the car, and measured the brain activity during actual driving. A purpose in this experiment was to measure and analyze the brain activity 
during actual driving to compare results between virtual and actual results. As a result, there were significant differences at similar regions [7][8]. In addition, we measured the brain activity of frontal lobe, which is related to behavioral decisionmaking, during car driving in different experimental design from previous one to verify previous results [9][10].

It is well known that higher order processing, such as memory, judgment, reasoning, etc. is done in the frontal lobe [11]. Human recognize the various information of car surrounding situation and perform car driving movement. We tried to elucidate the mechanism of information processing of the brain by analyzing data about human brain activity during car driving. Measuring of the brain activity by using NIRS is always affected by human body movement. In this paper, we tried to measure the brain activity during car driving and perform detailed analysis about human movement of car driving. Also, the goal of this study is to find a way to apply this result to new assist system.

\section{EXPERIMENT}

\section{A. Brain activity on virtual driving}

1) Measuring the Brain activity when subjects saw the driving movie.

We performed measurement which was under the virtual environment before performing measuring of actual car driving. And we confirmed the possibility of brain activity measurement. Experimental method was as follows.

The movie is included two scenes at a T-junction in which it must be decided either to turn to the right or left. In the second scene, there is a road sign with directions. We used nine kinds of movies in about one minute(Fig.1). Before showing the movie, subjects were given directions to turn to the right or left at the first T-junction. They were also taught the place which was on the road sign at the second T-Junction. They had to decide the direction when they looked at the road sign. They were asked to push a button when they realized the direction in which they were to turn.

The subjects for this experiment were eight males who were right handed. They were asked to read and sign an informed consent regarding the experiment.

An NIRS (Hitachi Medical Corp ETG-100) with 24 channels (sampling frequency $10 \mathrm{~Hz}$ ) was used to record the density of oxygenated hemoglobin (oxy hemoglobin) and deoxygenated hemoglobin (de-oxy hemoglobin) in the frontal cortex area.

2) Measurements of the brain activity when subjects performed the movement which was imitated the steering.

In this experiment, measuring was performed by NIRS, made by SHIMADZU Co. Ltd with $44 \mathrm{ch}$. Five subjects were healthy males in their 20s, right handed with a good driving history. They were asked to read and sign an informed consent regarding the experiment.

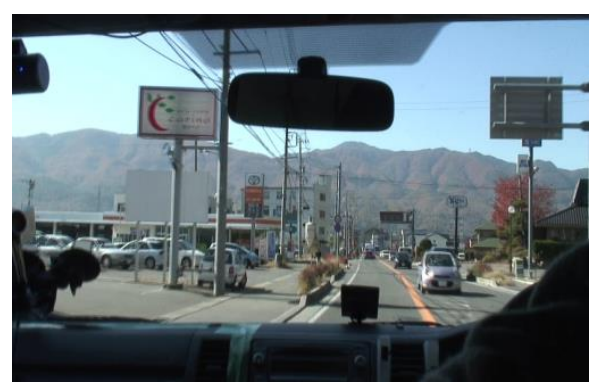

Fig.1. Perspective of the video which was used in the experiment

The subject was asked to perform simulated car driving, moving their hand in circles as if using a steering wheel. A PC mouse on the table was used to simulate handling a wheel, and NIRS (near-infrared spectroscopy) to monitor oxygen content change in the subjects' brain. NIRS irradiation was performed to measure brain activities when the subject sitting on a chair make a drawing circle line of the right or left hand. Also, we set the experimental condition which was the direction of clockwise or counterclockwise. The part of measurement was the frontal lobe. The subject was asked to draw on the table a circle $30 \mathrm{~cm}$ in diameter five times consecutively, spending four seconds per a circle. The time design was rest (10 seconds at least) - task (20 seconds) - rest (10 seconds) - close rest.

\section{B. Brain activity during actual car driving}

1) Measuring the brain activity when subjects drove on actual car

In general roads, experiments were performed by taking NIRS in the car, and measuring the brain activity when car driven by subjects was went through two different intersections. Six subjects were a healthy male in their 20s, right handed with a good driving history. They were asked to read and sign an informed consent regarding the experiment. In all experiments, measuring was performed by f-NIRS (Functional Near Infrared Spectroscopy), made by SHIMADZU Co. Ltd [11].

Subjects took a rest during 10 seconds at least with their eye close before driving task and they drove a car during about 600 seconds. Finally, subject closed their eyes for 10 seconds again after task. Then, the brain activity was recorded from the first eyes-closed rest to the last eyes. Subjects were given directions to turn to the right or left at the first $\mathrm{T}$-junction during driving task. They were also taught the place which was on the road sign at the second $\mathrm{T}$-junction. And, they were given the place where they have to go to. So, they had to decide the direction when they looked at the road sign.

A trigger pulse was emitted on stop lines at T-Junctions to use as a measuring stick for the analysis. Also, we recorded movie during the experiment from a car with a video camera aimed toward the direction of movement (Figure. 1). Recorded movies were used to exempt measurement result including disturbances, such as foot passengers and oncoming cars, from analysis. Figure. 2 and Figure. 3 shows one sample of $\mathrm{T}$ junction. 


\section{2) Verification Experiment}

To conduct verification for experimental results in previous experiment, we performed additional experiment which was

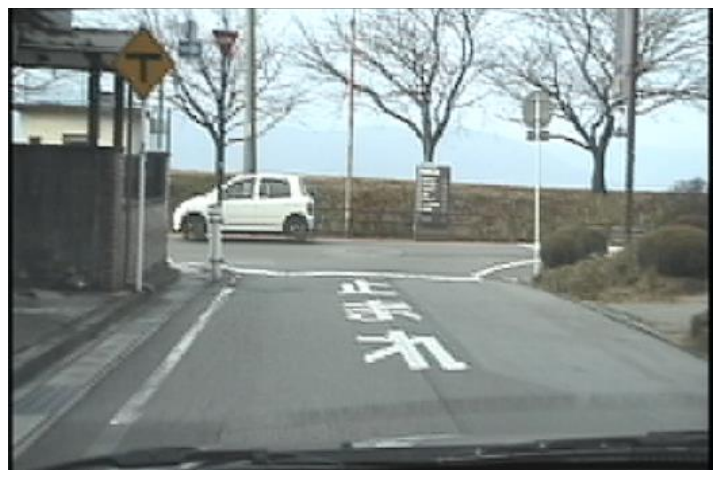

Fig.2. Sample of first T-Junction

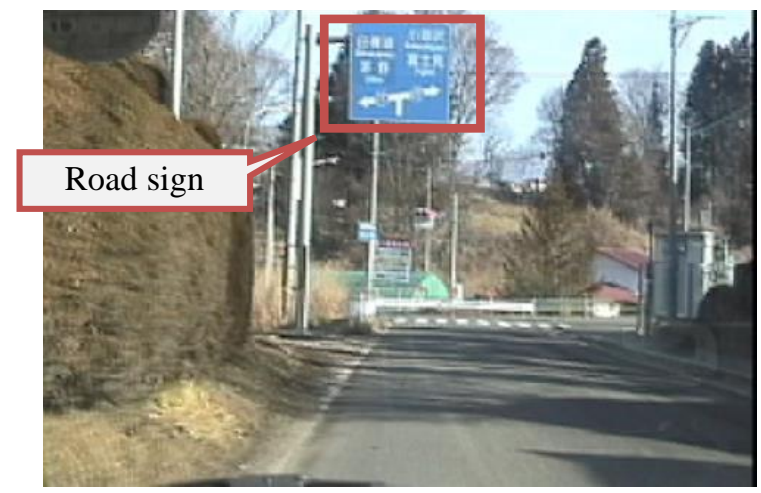

Fig.3. Sample of second T-Junction

achieved in a similar way. In this experiment, experimental course was different from previous one. While previous one was included two T- junctions in which there was road sign at second one and not at first one per a measurement, there were multiple T-junctions.

Scenes of turning at the T-junction were recorded on the movie. There were nine scenes in movies. In five scenes, the road sign was contained. In four scenes, the road sign was not contained.

Subjects were twelve males who were all right-handed. They drove a car during about 20 minutes after a rest during 10 seconds at least with their eyes close. Subjects were enlightened about turning direction and the place on which road signs was at T-junction during measurement. And, they arbitrarily decided the direction to turn when they confirmed road signs. Also, a trigger pulse was emitted in the same way.

\section{3) Detailed analysis based on driving behavior}

In this analysis, we focused on the movement of subjects during car driving. We verified that there are no relationship between decision-making of the direction and movement. We have attention to the movement of subjects arms and ocular. In previous research we performed, stop line at $\mathrm{T}$-junction was used as a trigger. But, brain activity in T-junction involved movement task such as turning steering wheel, changing neck direction, hitting the brake. So, it is thought that brain activity derived from cognitive tasks was overwritten with brain activity due to movement tasks. Therefore, we tried to analyze brain activity on the basis of ocular motions to examine significant differences with cognitive tasks.

\section{EXPERIMENTAL RESULTS}

\section{A. Brain activity on virtual driving}

1) Measuring the Brain activity when subjects saw the driving movie.

On the whole, the variation in de-oxy hemoglobin was smaller than in the oxy hemoglobin. It is considered that increasing oxy hemoglobin was caused by brain activity. Also, there was a great increase in channel 18 (around \#10 area of the dorsolateral prefrontal cortex of the right hemisphere). This might be the variation based on the spatial perceptions.

Next, differences were investigated concerning the subject's brain activity. As the first case, it was when the vision was directed after having been told the direction. As the Second, it was when the vision was directed after having been decided the direction under the road sign. We were trying to find a brain region involved in decision making that determines the direction by comparing these brain activity data in statistical analysis[12]. The experiment has been performed under the same conditions in any task without difference in instruction and difference between left and right. Therefore, the difference in instructions and difference between left and right and has appeared as a test result.

2) Measurements of the brain activity when subjects performed the movement which was imitated the steering.

In previous experiment, significant difference appeared due to the difference in the direction to turn left and right. In this experiment, we use PC mouse because, we confirm this results were due to the difference of the left and right rather than by the operation of the steering wheel. During the motion, the increase of oxy hemoglobin density of the brain was found in all subjects. The different regions of the brain were observed to be active, depending on the individual. The subjects were to be observed 1) on starting, and 2) 3-5 seconds after starting moving their 3) right hand 4) left hand 5)clockwise 6)counterclockwise.

We perform one sample t-test to analysis. Sample of t-test were oxy-hemoglobin at starting task and average of the average value of oxy-hemoglobin from 3 second to 5 second seconds after the start of the task.

Although some individual variation existed, the result showed the significant differences and some characteristic patterns. This pattern was increasing oxy hemoglobin density on starting task and decreasing oxy hemoglobin after end of task. The obtained patterns are shown as follows. Regardless of 1), 2), 3) and 4) above, the change in the oxy hemoglobin density of the brain was seen within the significant difference level 5\% or less in the three individuals out of all five subjects. The part was the adjacent part both of left pre-motor area and of left prefrontal cortex. Especially, in the adjacent part of prefrontal cortex a number of significant differences were seen among in four out of five subjects. Next more emphasis was put on the rotation direction: 5) clockwise or 6) counterclockwise. No large density change was found in the 
brain with all the subjects employing 6). Figure.4 shown the brain regions which were observed the statistical significant difference in each sample data.

In the Figure.4, brain regions which were draw by red. It is well known that in the outside prefrontal cortex higher order processing is done such as of behavior control. It is inferred that the pre- motor area was activated when the subjects moved the hand in the way stated above because the pre-motor area is responsible for behavior control, for transforming visual information, and for generating neural impulses controlling.

Subjects performed experiments in random order experimental conditions. Therefore it is not able to consider that experimental time and sequence effect of experimental conditions.

\section{B. Brain activity during actual car driving}

1) Measuring the brain activity when subjects drove on actual car

At the first, Hb-oxy was increased in overall frontal lobe after start of operation. This tendency was common among subjects. After that, Hb-oxy was decreased as subjects adjusted to driving the car. This meant that the brain activity changed from collective to local activities.

We performed one sample t-test as similar as previous virtual experiment. Fig. 5 and Fig.6 is shown the brain region where the statistical significant difference was seen.

\section{2) Verification Experiment}

Various tendencies among individuals were observed in comparison with results in result of actual car driving.

However, there were tendency that oxy-Hb was increased when car turned left or right at T-junctions and oxy-Hb was decreased during going straight.

Analysis method was the same as previous one. Though Gaps were shown regions at which there were significant differences, there were significant differences in \# 46 and premotor regions which was surrounded by red circles, too (Fig. 5 and Fig.6). In the analysis, measurement results including disturbance at $\mathrm{T}$-junctions were excluded as analysis object. The statistical significant difference was seen in the region like the previous experiments.

\section{3) Detailed analysis based on driving behavior}

The analysis was performed one-sample t-test within the significant difference level $5 \%$ or less between brain activity before and after looking at road sign. Each of sample data with respect to each direction which subject had to go at next Tjunction. As a consequence of analysis, there were significant differences at interior front gyrus of frontal lobe of left hemisphere without reference of direction (Fig. 8 and Fig. 9).

Also, we paid the attention to the movement of subjects for driving and performed one-sample t-test on the brain activity data. There are three analysis points which are the change of eyes vertical movement when subjects looked to road sign, the change of neck horizontal movement when subjects seen around situation and the change of hand movement when subjects turn a steering wheel. Figure was shown the collection method of the brain activity data to use for one sample t-test.

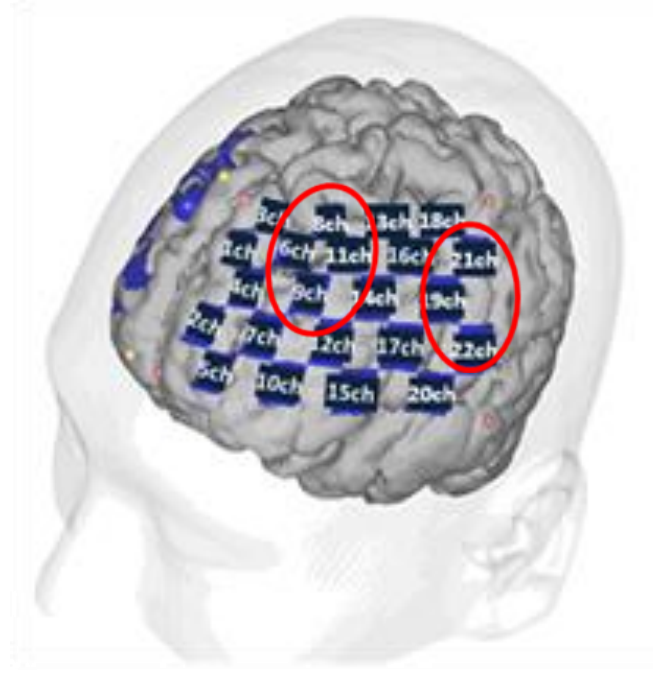

Fig.4. Brain activity of the statistical significant different.

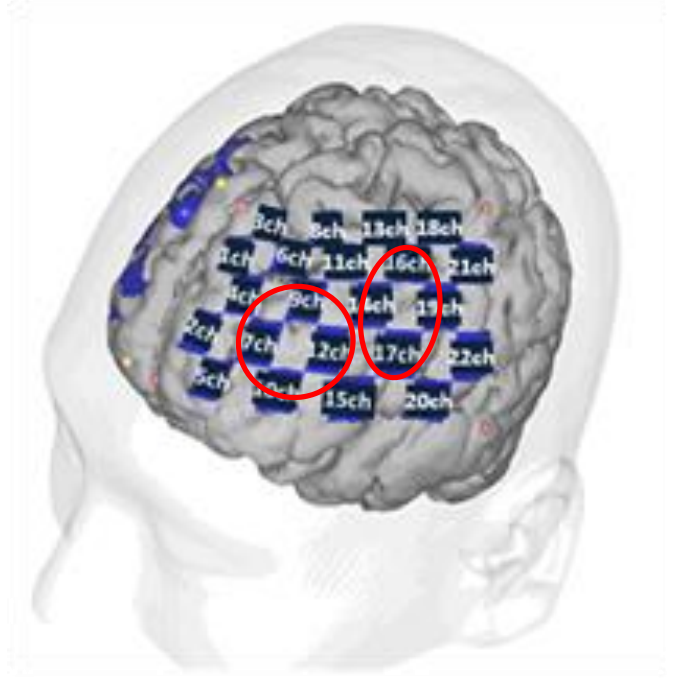

Fig.5. Significant differences when subjects turn left. 


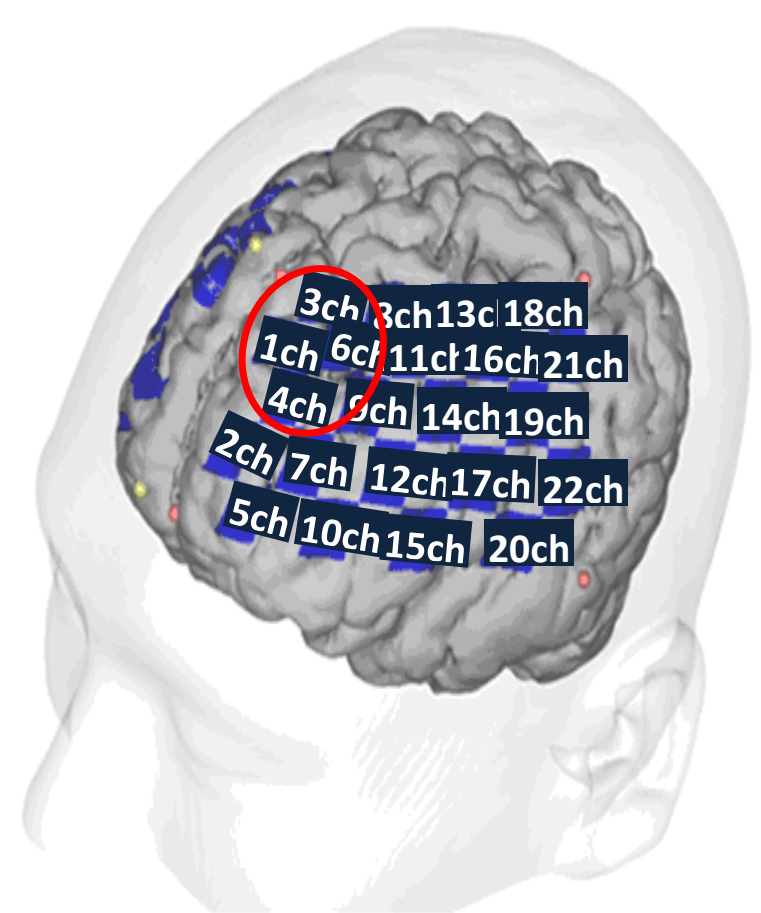

Fig.6. Significant differences when subjects turn right.

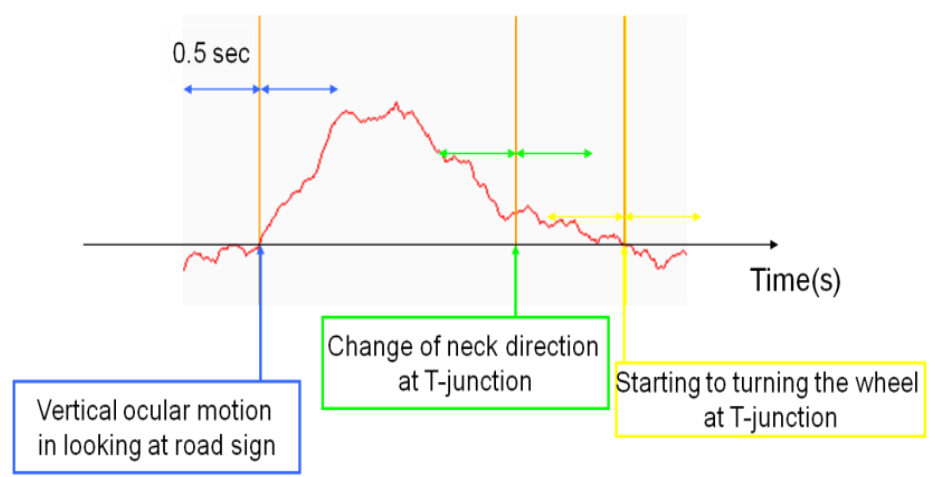

Fig.7. (a) Analysis method

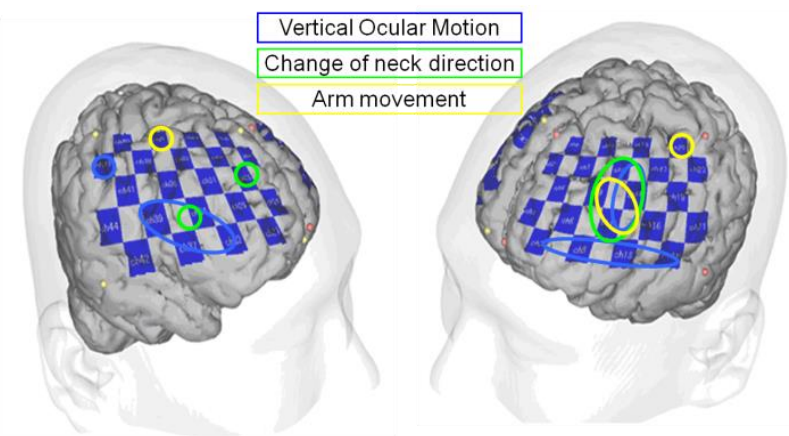

Fig.8. (b) Detail analysis when subjects turn left

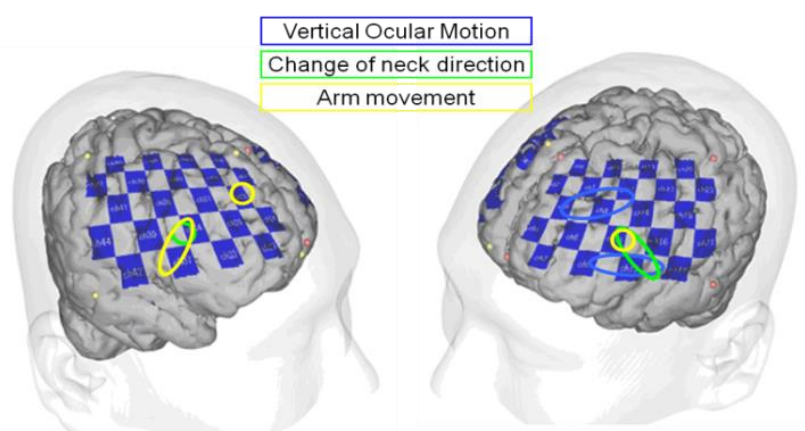

Fig.9. (c) Detail analysis when subjects turn right

\section{CONCLUSION}

The hemoglobin density change of the human subjects' frontal lobe was partly observed in the experiments we designed, where three kinds of tasks were performed to analyze human brain activity from the view point of spatial perception.

The NIRS measures of hemoglobin variation in the channels suggested that human behavioral decision-making of different types could cause different brain activities as we saw in the tasks: 1) taking a given direction at the first T-junction, 2) taking a self-chosen direction on a road sign at the second Tjunction and 3) turning the wheel or not. Some significant differences (paired t test) on NIRS's oxy-hemoglobin and less interrelated results between "pushing a button" and brain activity at the second T-junction are obtained.

Behavior related to the driving of the subject served to be the same condition as much as possible. It was not to use brain activity data when subjects performed different operation according to the situation of traffic. We think that differences in instruction and the difference between the turning left and right are reflected in the results of t-test.

In the analysis, t-test was performed on the condition that sample time length is 4 seconds or 1 second. In the general, it was said that hemoglobin density changes after a few seconds from being given stimulus. In the case of driving, subjects have to process various information and perform to operate car driving. If sample time length is long, it means that results of ttest include various information. By using the data of 1 second, we think that the target of t-test was limited only to the one brain activity.

In previous study, we performed one sample t-test above condition too, because we verify the results of the previous and this study.

Furthermore, experimental results indicated that with the subjects moving their hand in circle, regardless of right or left, 1) the same response was observed in the prefrontal cortex and premotor area, and 2) different patterns of brain activities generated by moving either hand clockwise or counterclockwise. 
The regions observed were only those with the 5\% and less significance level. Possible extensions could be applied to other regions with the $10 \%$ and less significance level for the future study. With a larger number of subjects, brain activity patterns need to be made clear. In addition, it is thought to take particular note of participation concerning working memory when car is driven.

Furthermore, it was found that there were significant differences around \#44-45 area. It is well known that this region is corresponding to language area. So, it is thought that subjects look at road map to determine direction that they have to go according to word described in road sign.

From results of these experiments, there was significant difference around working memory. So, experiments focusing on relationship turning wheel and working memory will be performed. On the other hand, experiments as to actual driving were required a broad range of perception and information processing. Especially, subjects had to determine behaves depending on various information at $\mathrm{T}$-junctions, that is, the color of the traffic light, presence or absence foot passengers and so on. And so, we plan to perform more static experiments. we attention to differences on the basis of turning direction and dominant hand. In addition, we will conduct the experiments in which subjects were narrowed down to left-handedness. Furthermore, researches into other human brain activities than spatial perception are to be necessary with accumulated data from fMRI (functional magnetic resonance imaging), EEG (Electroencephalogram), etc.

When compared virtual result to actual ones, there were significant differences around \#46 area in both experiments, which were performed in virtual and actual condition, as a common result. It is thought that this result is due to activities of working memory because subjects must to recall memories of movements required for car driving and turning steering wheel. Conversely, there were significant differences around \#10 in virtual experiments and around premotor area in actual driving, respectively. In the virtual case, it is thought to result from inhabitation of task without movement. In the actual case, subjects had to perceive space information in real time. So, it is considered that there were significant differences around premotor area because they always ready up to manipulate steering wheel.

In addition, we performed one sample t-test based on subjects' movement as detail analysis. Particularly, we paid the attention to the movement of the neck which confirmed road reputation, the lateral movement of the neck in the crossing, movement for steering wheel operation of the hand and performed one sample t-test. As a result, there is the significant difference on the difference brain region in each t-test analysis. Also, these results were difference from previous results that performed one sample t-test by the brain activity data when subjects were taught the direction or names of places. All the viewpoints are human movement about driving car in the Tjunction when we analyzed it in detail. Thus, we think that decision making appears for the result of the expression point of the statistical significant difference not developing by movement when I decide a direction.

\section{FUTURE WORK}

As a future plan, we aim to apply these results to assistive human interface. As a matter of course, we plan to performed additional experiments including the verification of these results. And final purpose is to develop a new system for manipulating wheelchair and information presentation system to assist recognition of information including spatial one during car driving. Recently, some systems to assist the driver by using a lot of sensor have been developed in some car company. We think that these results are considered to develop some assist systems. For example, we think that the system which prevents mistakes of human decision and driving assist system to using BMI. Therefore, it is necessary to measure more the brain activity data and make the database.

\section{REFERENCES}

[1] M.J. Kahana, R. Sekuler, J.B. Caplan, M. Kirschen, and J.R. Madsen: "Human theta oscillations exhibit task dependence during virtual maze navigation.”, Nature, 1999, 399, pp. 781-784.

[2] N. Nishiyama and Y. Yamaguchi: "Human EEG theta in the spatial recognition task", Proceedings of 5th World Multiconf. On Systemics, Cybernetics and Informatics (SCI 2001), Proc. 7th Int. Conf. on Informatiofn Systems, Analysis and Synthesis (ISAS 2001), pp. 497-500 (2001).

[3] E.A. Maguire, N. Burgess, J.G. Donnett, R.S.J. Frackowiak, C.D. Frith, and J.O' Keefe: "Knowing Where and Getting There: A Human Navigation Network," vol. 280 Science 8/may/1998.

[4] Chin-Teng Lin, Shi-An Chen, Tien-Ting Chiu, Hong-Zhang Lin, and LiWei Ko: "Spatial and temporal EEG dynamics of dual-task driving performance." Journal of NeuroEngineering and Rehabilitation, vol. 811,2011

[5] S. Shimizu, N. Hirai, F. Miwakeichi, and et al: "Fundamental Study for Relationship between Cognitive task and Brain Activity during Car Driving," Proc. the 13th International Conference on Human-Computer Interaction, (San Diego, CA, USA, 2009), Springer Berlin / Heidelberg, 434-440.

[6] N. Takahashi, S. Shimizu,Y. Hirata, H. Nara, F. Miwakeichi, N. Hirai, S. Kikuchi, E. Watanabe, and S. Kato: "Fundamental Study for a New Assistive System during Car Driving," Proc. International Conference on Robotics and Biomimetics, 2010, China.

[7] N. Takahashi, S. Shimizu, Y. Hirata, H. Nara, H. Inoue, N. Hirai, S. Kikuchi, E. Watanabe, and S. Kato,"Basic study of Analysis of Human Brain Activities during Car Driving," the 14th International Conferrence on Human-Computer Interaction, 2011, Orlando, Florida, USA.

[8] S. Shimizu, N. Takahashi, H. Nara, H. Inoue, and Y. Hirata "Fundamental Study for Human Brain Activity Based on the Spatial Cognitive Task," the 2011 Internatinal Conference on Brain Informatics-BI 2011, China.

[9] S. Shimizu, H. Nara, N. Takahashi, H. Inoue and, Y. Hirata,"Basic Study for Human Brain Activity Based on the Spatial Cognitive Task," The Third International Conference on Advanced Cognitive Techonologies and Applications, 2011, Italy.

[10] J. Cockburn: "Task interruption in prospective memory: "A frontal lobe function?." Cortex, vol. 31, 1995, pp. 87- 97.

[11] E. Watanabe, Y. Yamashita, Y. Ito and, H. Koizumi, "Non-invasive functional mapping with multi-channel near infra-red spectroscopic topography in humans," Heurosci Lett 1996, Feb 16, 205(1), 41-4. 